\title{
DOKTRINÄRE AUSWIRKUNGEN DER ERSTEN INTERNATIONALE IN DER SCHWEIZ
}

Wenn man die Doktrinen, welche die Internationale in der Schweiz gefördert und entwickelt hat, überblicken und würdigen will, muss man sich der besonderen Schwierigkeiten dieses Unterfangens bewusst sein, Schwierigkeiten der Grenzziehung, der Bewertung und der Auswahl. Eine verwirrende Fülle von Lehrmeinungen begegnet uns. Es gibt keine zuverlässigen Kriterien, nach denen sie sich in wichtig und unwichtig scheiden lassen, oder nach denen eine über alle Zweifel erhabene zeitliche Grenze zu ziehen wäre. Die Beschränkung auf zwei führende Doktrinäre oder Schulen, auf Johann Philipp Becker und die Jurassier, hat mehr den Sinn einer typologischen Auslese als den einer Bewertung. Becker erscheint nicht etwa als Chefideologe der Internationale, sondern als typisch für den Gegensatz zur jurassischen Schule, als der repräsentativste Vertreter der Anliegen des Londoner Generalrats, als ein Mann, der sich in dessen Gedankenwelt am intensivsten eingelebt, und der kraft seiner schriftstellerischen und brieflichen Tätigkeit einen überdurchschnittlich grossen Einfluss auf die Schweizer Sektionen der Internationale ausgeübt hat. Als Vertreter einer „Dritten Kraft”, die so stark ist, dass die Internationale gezwungen ist, unablässig mit ihr Kompromisse einzugehen, wäre die ältere Generation zu betrachten, ein Bürkli und Coullery. Sie bleiben aber unberücksichtigt, weil sie - im Gegensatz zu Becker - ihrer „vor-internationalen” Gedankenwelt treu bleiben. Uebergangen wird auch die jüngere Generation, etwa ein Hermann Greulich. Obschon sich die Ansätze zu einem eigenen Lehrgebäude schon in den Siebzigerjahren recht deutlich zeigen, so reift doch seine Doktrin als Ganzes erst in einer späteren Zeit aus und gehört darum nicht mehr in den Zeitraum der Ersten Internationale. ${ }^{1}$

${ }^{1}$ Die folgenden Ausführungen fassen einige Ausschnitte aus dem demnächst in Bern beim Verlag Francke erscheinenden Werke Die Arbeiter in der Schweiz (Soziale Lage, Organisation, Verhältnis zu Arbeitgeber und Staat im 19. Jahrhundert) zusammen. 
Johann Philipp Becker ist vor allem als unermüdlicher Agitator und Organisator, als eine Art Berufsrevolutionär bekannt. Sein grosses schriftstellerisches Werk ist heute, ausser etwa seine im Vorboten erschienenen Aufsätze, ganz vergessen. Obwohl Gridazzi Becker als bedeutungsvollem Träger "sozialistischer Ideen in der Schweiz" eine umfangreiche Würdigung widmet, gesteht er freimütig, dieser sei als Theoretiker uninteressant. ${ }^{1}$ Aber obgleich Becker - ähnlich wie Bürkli und Treichler - nicht den Ruhm eines selbständigen sozialistischen Denkers beanspruchen darf, so besitzt doch gerade sein organisatorisches Werk einen lehrhaften Charakter. Becker ist als Agitator der erste konsequent sozialistische Erzieher der schweizerischen Arbeiterschaft. Im grösseren Rahmen der deutschen Sprachsektion vermittelt er auch den deutsch sprechenden Arbeitern unseres Landes die Doktrin der Internationale.

Doch welcher der anfänglich in ihr so bunt durcheinander wogenden Lebrmeinungen gibt Becker den Vorzug? Bei der Antwort auf diese Frage scheiden sich die Geister. Die Biographen aus dem ostdeutschen Bereich, Dlubek, Engelberg und Trübner, erblicken in Becker mehr oder weniger ausschliesslich und von Anfang an einen Vertreter Marxens. Dlubek und Trübner streiten zwar Beckers nachweisbare Anleihen bei Lassalle und Proudhon nicht ab. Trübner gibt zu, unter dem Einfluss von Proudhon sei Becker versöhnlich geworden und habe z.B. in der Frage der Produktionsgenossenschaften geradezu eine nicht marxistische Linie vertreten. ${ }^{2}$ Bei Dlubek dagegen erscheint z.B. Beckers Anlehnung an Lassalle nicht als Abweichung, sondern als marxistische Interpretation. Becker folge Lassalle nur insofern, als dieser das Proletariat klassenmässig organisiert und sich mithin auf einer marxistischen Linie befunden habe. ${ }^{3}$ Engelberg sagt kurz und bündig, Becker habe seine Ansicht stets derjenigen von Marx hintangesetzt. ${ }^{4}$ Dass Braunthal Becker als „,begeisterten Anhänger der

\footnotetext{
${ }^{1}$ Mario Gridazzi, Die Entwicklung der sozialistischen Ideen in der Schweiz bis zum Ausbruch des Weltkrieges, Zürich 1935, S. 138-163, 141.

2 Georg Trübner, Johann Philipp Becker, Ein Leben für die Freiheit (1809-1886), Hab. schrift Jena I956, Masch. schrift.

${ }^{3}$ Rolf Dlubek, Johann Philipp Becker, vom radikalen Demokraten zum Mitstreiter von Marx und Engcls in der Ersten Internationale, Diss. phil. I Berlin 1964, Masch. schrift.

${ }^{4}$ Ernst Engelberg, Fragen der Demokratie und des Sozialismus in der I. Internationale. Einführung zur originalgetreuen Reproduktion des „Vorboten”, Berlin-Zürich 1963 , S. 59 .
} 
Ideen von Garibaldi wie Lassalles, und von Marx wie Bakunin"'1 bezeichnet, reizt Engelberg zum Widerspruch. Ahnlich müsste er auch auf die neueste Darstellung R. P. Morgans reagieren, für den Becker - in Anlehnung an ein Wort von Engels - ganz einfach ein Mann der Aktion ist, "unreflecting", so dass er als urtümlicher Freischärler in der Zeit der Internationale oft wie ein Fremdkörper wirke. ${ }^{2}$ Einer andersartigen Einseitigkeit macht sich Gridazzi schuldig. Nach ihm wäre Becker überhaupt nicht als marxistischer Sozialist anzusprechen, sondern höchstens als eine Kreuzung des französischen Frühsozialismus mit Lassalle und Marx. ${ }^{3}$ Er verbinde eine idealistische Weltanschauung mit einer materialistischen Geschichtsauffassung. ${ }^{4}$ Es geht indessen nicht an, in Becker einfach einen Aufklärer zu sehen, der die oekonomischen Probleme in den Hintergrund rücke und einen metaphysischen Glauben an die Ideenwelt besitze. ${ }^{5}$ Ebensowenig darf man Becker zum Reformisten stempeln. ${ }^{6}$

Angesichts dieser Interpretationsunterschiede ist es nicht einfach $z u$ bestimmen, welches der echte Kern der Beckerschen Lehre ist. Becker teilt mit den meisten Agitatoren, so etwa auch mit Wilhelm Liebknecht, die Eigenschaft des Eklektikers. In dieser Feststellung liegt kein Vorwurf. Der Eklektiker ist weder eine Windfahne noch ein gedankenloser Nachbeter fremden Geistesgutes. Sein Wesen besteht vielmehr darin, dass er eine einmal gefasste Ueberzeugung als ergänzungswürdig betrachtet. Darum versucht er, neu gewonnene Ansichten mit seinen bisherigen Anschauungen jeweilen zu einer neuen Einheit zu verschmelzen. Das gibt im Grunde auch Trübner zu, wenn er betont, der Vorbote sei der Spiegel für Beckers allmähliche Wandlung vom Kosmopoliten zum wissenschaftlichen Sozialisten.7 Gerade deshalb erscheint es gefährlich, wenn Dlubek in Beckers geistiger Entwicklung das Jahr I 860 als entscheidende Zäsur bezeichnet, weil Becker damals zum ersten Male mit Marx in brieflichen Kontakt getreten sei. ${ }^{8}$ Es kann hier gerade nur erwähnt werden, dass

\footnotetext{
1 Julius Braunthal, Geschichte der Internationale, Bd. I, Hannover 1961, S. 124.

2 R. P. Morgan, The German Social Democrats and the First International 1864-72, Cambridge Univ. Press 1965.

- Gridazzi, Die Entwicklung der sozialistischen Ideen, S. I44ff.

4 Ebenda, S. 147, 150.

- Ebenda, S. I44f., I gof. Gridazzi verwechselt die angebliche Metaphysik Beckets mit dem Marx inhärenten Glauben an das Proletariat als dem Träger der absoluten Wahrheit. - Ebenda, S. I4sf. Gridazzi stützt sich auf eine Kautsky-Interpretation der Inauguraladresse aus dem Jahre 1922, aus der hervorgehe, dass die Reform ebenso zum Marxismus gehöre wie die Revolution, ohne zu bedenken, dass die damalige Stellungsnahme Kautskys nur als Abwehr gegenüber dem Bolschewismus verständlich ist.

- Trübner, J. P. Becker, S. 335.

8 Dlubek, J. P. Becker, Kap, V, S. 247 ff.
} 
sich die entscheidenden Ansätze zu seiner späteren Grundhaltung schon während der Revolution von 1848 herausbilden. Unter dem Einfluss Galeers und Willichs bekehrt sich Becker damals zu einem Kosmopoliten mit sozialer Färbung. Er erlebt das Proletariat als Träger der sozialen Revolution und Schöpfer der roten Republik. Als Mitkämpfer von Engels deutet er die badische Revolution von I 849 schon als eine Art Klassenkampf. Dagegen steht er in bewusstem Gegensatz zu den Vertretern des Kommunistenbundes in Genf. ${ }^{1}$

So geht also Becker von zwei Konstanten aus, die sich in groben Umrissen gleichsam als „Internationalismus” und „Sozialismus” bezeichnen lassen. Sie präsentieren sich aber durchwegs noch als Gefühlswerte und ermangeln der klaren Konturen und der inneren Systematik, die man bei einer Doktrin voraussetzt. Davon zeugt in besonderer Weise etwa Beckers zwischen I850 und I864 entstandenes Schrifttum. Seine umfangreichen Werke "Wie und wann"2 und die „Psalmen in Reimform"' stellen nur Variationen dieser beiden gleichsam axiomatischen Grundwerte dar. In ihnen äussert sich ein dumpfes Erlösungsstreben gegenüber bevormundenden Kräften, eine Anklage an die Mächtigen dieser Welt, denen man die Maske des Eigennutzes vom Gesicht reissen muss, und die Sehnsucht nach Ueberwindung der persönlichen und nationalen Beschränktheit. Bezeichnend für dieses Sinnen und Trachten ist der Schluss eines Bekenntnisses, das Becker in Zusammenhang mit einem 18,6 verfassten Curriculum vitae ablegt. ${ }^{4}$ Er wünscht Gleichberechtigung aller Menschen nach Massgabe ihrer intellektuellen Kräfte, Fähigkeiten und ihrer Tatkraft. Als Ziel des Sozialismus bezeichnet er den gemeinschaftlichen, in jeder Richtung zu veredelnden Genuss aller Lebensgüter auf dem Wege permanenter Revolution und die organisch gegliederte Einheit der Menschen. Unter ähnlichen Vorzeichen steht auch Beckers politische Aktivität zu Beginn der Sechzigerjahre, nachdem er von seinem Freischarenabenteuer bei Garibaldi heimgekehrt ist. Bevor noch die Internationale ihren Ruf hat erschallen lassen, sammelt er im Geiste Galeers die „werktätige Klasse” in Genf zum Klassenkampf. Mit dem „Deutschen

\footnotetext{
${ }^{1}$ Die Behauptung Dlubeks, op. cit., S. 279, Becker habe in Genf das Erbe des Bundes der Kommunisten weitergeführt, ist falsch.

2 Wie und wann? Ein ernstes Wort über die Fragen und Aufgaben der Zeit. Genf, London, Manchester I 862.

3 Psalmen in Reimform. Neue Stunden der Andacht. Das Zeitgebrechen, blossgelegt durch strikte Schlussfolgerungen aus den überlieferten Anschauungen und Einrichtungen in religiöser, politischer, ethischer und sozialer Beziehung. Genf I875. Dieses Werk ist I $857-60$ konzipiert worden.

4 N. Rjazanow, Zur Biographie von Johann Philipp Becker. Sein Curriculum vitae bis 1856, in: Archiv für die Geschichte des Sozialismus und der Arbeiterbewegung, 1914, S. 318.
} 
Republikanischen Volksbund”, der sich Lassalles „Allgemeinem Deutschen Arbeiterverein" anschliesst, sucht er den Grund einer neuen Völkerverbrüderung zu legen. Morgan nennt diese letzte Absicht mit Recht ,unseasonable and unpracticable echoes of 1848 ". ${ }^{1}$ Wir möchten es lieber so sagen: sie stehen mit den sozialistisch-kosmopolitischen Vorstellungen von 1848 in natürlicher Beziehung, weil Becker sich noch nicht über diese hinaus gewagt hat.

Erst in der Auseinandersetzung mit führenden revolutionären Geistern der Ersten Internationale verwandeln sich diese stimmungsmässigen Vorstellungsgebalte aus ihrem gefühlsmässigen in einen rationalen Aggregatzustand. Erst in der Begegnung mit Lassalle, Proudhon, Bakunin und Marx entwickeln sich also die Axiome des Kosmopolitismus und der Emanzipation der Arbeiterklasse zu einer mehr oder weniger geschlossenen Doktrin. Aber dieser Uebergang ist nun wesentlich bestimmt durch Beckers pragmatische Haltung. Als urtümlicher Revolutionär, als Organisator, als Taktiker, als Genfer Lokalpolitiker, als Leiter der deutschen Sektionsgruppe besitzt Becker stets wechselnde geistige Bedürfnisse. Darum ist er so vielen und verschiedenartigen Einflüssen offen. Er nimmt etwas an, verwirft es aber hernach nur halb und vermengt es wiederum mit etwas Neuem! Als 48er Revolutionär wird er von Lassalles Doktrin angezogen. Als gewerkschaftlicher Pionier folgt er vorübergehend Proudhons Spuren. Bakunins Revolutionsideologie dient ihm zeitweise als Gegengewicht gegen die kleinliche Lokalpolitik der Genfer Uhrmacher. Erst als die Genfer Lokalgrössen in ihrem Kampf gegen Bakunin mit Marx ein Bündnis auf Gedeih und Verderb eingehen, lehnt er sich definitiv an dessen Doktrin an. ${ }^{2}$ Nun verfolgt er Bakunin und seine Lehre, wie dies nur ein Apostat tun kann. Nachdem Beckers enge Freundschaft mit Bakunin und seine Opposition gegen die Gründung der Eisenacher Partei ihn von Marx entfremdet hat, versucht er, sich nun durch doppelte Dienstfertigkeit bei den Londoner Dioskuren wieder in Gunst zu setzen. Er unterstützt Marxens Zuträger Utin bei der Sammlung des Klatsches, den dieser zur moralischen und politischen Aechtung Bakunins benötigt. Er hilft mit, die Freunde Bakunins aus der Redaktion der Egalité auszubooten und Utin an ihre Stelle zu placieren. ${ }^{3}$ Nach der Spaltung der „Fédération romande” im April r 870 unterstützt er die Genfer Lokalgrössen in ihrem Kampf gegen Bakunin und seine jurassischen Gesinnungsgenossen. Nach

${ }_{1}^{1}$ Morgan, German Social Democrats, S. 70.

2 Ueber Beckers enge Zusammenarbeit mit Bakunin in Genf vgl. Marc Vuilleumier, Bakounine, l'Alliance Internationale de la Démocratie socialiste et la Première Internationale à Genève (1868-69), in: Cahiers Vilfredo Pareto 1964, S. 51-94.

8 Näheres über diese Machtkämpfe im Gesamtwerk Die Arbeiter in der Schweiz. 
dessen Ausstossung aus der Internationale auf dem Kongress von Den Haag lässt er eine hasserfüllte, von persönlichen Ressentiments geladene Artikelserie gegen Bakunin in der Tagwacht erscheinen. ${ }^{1}$ Im September 1873 mobilisiert Becker, freilich erfolglos, den verunglückten internationalen Kongress in Genf als Gegenaktion gegen den erfolgreichen Gründungskongress der antiautoritären Internationale durch James Guillaume und seine Freunde, der in derselben Stadt abgehalten wird. Selbst nach dem Tode Bakunins fühlt er sich berufen, die Versöhnungsversuche zwischen den „Anti-Autoritären” und den Ueberresten der alten Internationale zu sabotieren, die unter dem Einfluss de Paepes, Vahlteichs und anderer am Berner Kongress der anti-autoritären Internationale im Oktober $r 876$ eingeleitet werden. ${ }^{2}$

In dieser pragmatischen Haltung liegt also die Wurzel seines Eklektizismus. Doch offenbart sich trotz allen Zickzacklinien in Beckers geistiger Entwicklung eine gewisse Konstante. Becker ist auf Lehren ansprechbar, in denen er seine axiomatischen Anliegen bestätigt, geklärt, erweitert und systematisiert findet. So gleicht sein Lehrgebäude in seiner letzten, auf dem Höhepunkt der Internationale erreichten Form einer Folge von Sedimenten. Es lässt sich deshalb am besten verstehen, wenn man sein Verhältnis zu seinen Lehrmeistern in der geschichtlichen Abfolge betrachtet.

In Lassalles Schriften findet Becker vorerst eine Bestätigung seiner Revolutionsideale von I 848. Lassalle erscheint ihm als der Fortsetzer des 1848 verlorenen Klassenkampfes. Becker bewundert ihn, weil er das damals noch kaum organisierte Proletariat zur geschlossenen Klassenpartei umformt und es damit instandsetzt, zum letzten Kampf gegen die Bourgeoisie aufzutreten. ${ }^{3}$ Im erstrebten deutschen Volksstaat sieht er das Ideal der roten Republik verwirklicht, das Becker I 848 vergeblich zu erreichen gesucht hatte. ${ }^{4}$ An Lassalle geht Becker

\footnotetext{
1 Beckers Angriffe auf Bakunin in der Tagwacht beginnen schon kurz nach der Spaltung der „Fédération romande” auf dem Kongress zu La Chaux-de-Fonds. Am 23. April 1870 erscheint z.B. in No. Io der Tagwacht eine sehr einseitige Darstellung dieses Kongresses. "Gewiss ist", steht dort zu lesen, „dass er [sc. Bakunin] als bezahlter Agent durch seine unklaren und unreifen Ideen gar nicht besser für den Despotismus wirken konnte"; vgl. auch No. 12 und 13 vom 7. und 14. Mai I 870. Die Artikelserie nach dem Haager Kongress setzt in No. 4I der Tagwacht vom 12. Oktober 1872 ein und endet erst in No. 12 vom 22. März 1873. Sie ist das Muster einer Verunglimpfung eines ehemaligen Freundes.

2 Man vergleiche z.B. seine zwiespältigen Artikel in der Tagwacht, No. 83 und 84 vom 18. und $2 \mathrm{x}$. Oktober 1876 .

8 Offener Brief an die Arbeiter über Schulze-Delitzsch und Lassalle, die Bourgeoisie und das Proletariat, Genf 1863 , S. 1, 3, 10, 43.

4 Ebenda, S. I r, 30.
} 
erstmals auf, dass der soziale Kampf wissenschaftlich begründet werden muss. Aber er versteht die gepriesene "Sozialwissenschaft" noch ausschliesslich von der Gefühlsseite her. Die Sozialwissenschaft soll „der Leucht-, Brenn-, und Haltpunkt aller Thätigkeit in Idealismus, Materialismus und Realismus” sein. „Es gilt die Bedürfnisse des Menschen in Familie, Gemeinde und Staat in Harmonie zu bringen, Politik, Religion und Moral in einheitliche Gedanken zu fassen, den Geist, das Gemüth und den Leib durch die innig vereinigten Leistungen der Wissenschaft, der Kunst und des Gewerbes zu befriedigen das Nützliche, Schöne und Angenehme für alle hinreichend zu erzeugen ...". ${ }^{1}$ Die Angelpunkte in Lassalles System, das eherne Lohngesetz und der Anspruch auf gerechten Arbeitsertrag, stellen aber für Becker nicht Resultate wissenschaftlicher Ueberlegungen dar, sondern isolierte Glaubenssätze. ${ }^{2}$ Es entspricht durchaus der eklektischen Natur Beckers, dass er später, nachdem er sich längst von Lassalle entfernt hat, dessen Lehre als gleich berechtigten Teil eines umfassenden Ganzen gelten lassen will und nur die förmliche Bildung einer Lassalleaner-Schule verurteilt. ${ }^{3}$ Er vergleicht den Sozialismus mit einem Tempel, der unter einem einheitlichen Dach verschiedene Lehrmeinungen ohne Scheidewände vereinigt. ${ }^{4}$ Denn sie alle sind letztlich nur Varianten der „von Ewigkeit her gewesenen und in Ewigkeit hin bleibenden Grundsätze". ${ }^{5}$ Damit verwirft Becker zum voraus Marxens Prinzip, die Internationale auf eine einzige, autoritär fixierte Lehrmeinung festzulegen. Für „Personenkult und Autoritätsglauben" hat er nur Spott und Verachtung übrig. ${ }^{6}$ Nur diese Haltung macht es erklärlich, dass er nach der Aneignung der Marxschen Gedankenwelt 1869 gleichzeitig in enge Beziehungen zu Bakunin tritt, ohne sich des Widerspruchs zwischen beiden Systemen offenbar klar bewusst zu werden.?

Im Gegensatz zu Lassalle und Marx dürfte Becker Proudbons Lehre kaum genau gekannt haben. Seine Verbindung mit ihm muss also auf die dominierende Stellung zurückgeführt werden, welche dessen französische und welschschweizerische Anhänger an den Kongressen von Genf und Lausanne einnehmen. Proudhon denkt, kurz gesagt, an die unbegrenzte Ausdehnung der Gegenseitigkeit und Zusammen-

1 Ebenda, S. I 8.

2 Ebenda, S. 28ff., $37 \mathrm{f}$.

3 Vorbote 1869, S. $65-70$.

4 Ebenda, S. 67.

5 Ebenda.

- Ebenda, S. 65.

7 Ueber das Verhältnis von Bakunin zu Becker vgl. auch N. Rjazanow, Bakuniana, in: Archiv für die Geschichte des Sozialismus und der Arbeiterbewegung, 1915, S. 182-189. 
arbeit. Der Sieg der beiden Prinzipien der „Mutualité” und „Coopération" in der oekonomischen Produktion, d.h. die demokratische Organisation des Kredits und des Warenaustausches, und ihre Uebertragung auf die politische Verfassung bilden also die einzige Voraussetzung für die Emanzipation der Arbeiterklasse. Während der Vorherrschaft der Proudhonisten erscheinen nun die Beckerschen Axiome unvermutet in neuer Beleuchtung. So tritt jetzt z.B. der Klassenkampf in den Hintergrund. ${ }^{1}$ Einzig die genossenschaftliche Arbeit im Sinne Proudhons vermag die Klassengegensätze aufzuheben. ${ }^{2}$ Die Produktionsgenossenschaften erscheinen jetzt geradezu als Mittel, „die alte Ordnung aus den Angeln zu erheben". 3 Becker interpretiert damals den zentralen Grundsatz der Internationale, die Ersetzung des Arbeitslohnes durch den Arbeitsertrag, rein proudhonistisch. ${ }^{4}$ Den internationalen Sozialismus, der nach Proudhon die bestehende Staatenwelt in eine Foederation von Genossenschaften verwandeln soll, erlebt Becker als „menschheitliche Demokratisation".5 Die Internationale wird noch ganz kosmopolitisch als Basis einer „europäischen Eidgenossenschaft" gedeutet.

Beckers Abkehr vom proudhonistischen System fällt äusserlich mit der Niederlage seiner Vertreter am Brüsseler Kongress zusammen.7 Die theoretischen Argumente, mit denen die Proudhonschen Produktionsgenossenschaften dort verurteilt werden, vor allem ihre Machtlosigkeit gegenüber dem Grossbetrieb, werden zusätzlich durch deprimierende Erfahrungen mit schweizerischen Produktionsgenossenschaften erhärtet. ${ }^{8}$ Damit verstärkt sich Beckers Tendenz, seine Axiome im Sinne Marxens umzudeuten. Als Becker 1860 erstmals brieflich mit Marx in Verbindung tritt, da versteht er sein Verhältnis zu jenem noch durchaus gefühlsmässig: „Durch unsere politischen und sozialen Standpunkte in Denkungs- und Handlungsweise müssten wir längst Freunde sein und werden es aus gleichen Stücken auch immer bleiben". ${ }^{9}$ Man kann den Moment, da Becker das Marx-

\footnotetext{
1 Vorbote 1866, S. 146, Diskussion über Streik. Das Bekenntnis zur Marxschen Formel von der Eroberung der politischen Macht wird freilich von Becker schon 1866 vereinzelt gebraucht, Vorbote, 1866, S. 88.

2 Ebenda, S. 26.

Ebenda, S. 3.

1 Ebenda, S. 146.

Ebenda, S. 15.

- Ebenda, S. 18, 55 .

7 Ebenda, 1868, S. 146ff.

B Ebenda, 1868, S. 166ff. Einige Produktionsgenossenschaften werden vor allem durch Boykotte der Lieferanten ruiniert.

- Becker an Marx, Paris 20. April 1860, Marx-Engels Nachlass D 230 (Photokopie), IISG Amsterdam.
} 
sche System sich rational aneignet, zeitlich nicht fixieren. Man kann diesen lange dauernden Prozess höchstens in einem allmählichen Stil- und Auffassungswandel im Spiegel des Vorboten verfolgen. An die Stelle des kosmopolitischen Ideals der menschheitlichen Demokratie tritt jetzt definitiv die Vorstellung des internationalen Sozialismus. Der Klassenkampf, den Becker bei Lassalle noch als Polarität zwischen der sozialdemokratischen und der Fortschrittspartei empfindet, wird nun erstmals oekonomisch gedeutet, als Folge der modernen Produktionsweise. So machen die bisherigen gefühlsmässigen Vorstellungen den Marxschen Theorien Platz, der Konzentration des Kapitals, der Proletarisierung der Mittelschichten, der zunehmenden Verelendung. ${ }^{1}$ Das vage bisherige Sozialmodell wird jetzt auf Marxens Zweiklassenantagonismus reduziert. Becker spricht jetzt von einer „Regressionsund einer Regenerationspartei”. ' Die Vorliebe für Genossenschaften und Gewerkschaften behält aber Becker bei. Doch erscheinen jetzt diese im Sinne Marx' als „Vorschulen der Partei”. Mit der „Rekrutenschule der Arbeitseinstellungen" beginnt die revolutionäre Arbeiterbewegung. „Denn die Arbeiterbewegung, wenn auch noch nicht überall zum allgemeinen Bewusstsein gekommen, ist die Revolution, und zwar zum universalsten und radikalsten Umschwung in der Weltgeschichte". ${ }^{3}$ Der emotionale Wissenschaftsbegriff, den wir in Beckers Schrift über Lassalle getroffen haben, wird jetzt ersetzt durch den Begriff des wissenschaftlichen Sozialismus. ${ }^{4}$ Jetzt schlüpft Becker also ganz in die Hülle des historischen Materialismus und bejaht die von Marx behauptete Zwangsläufigkeit des Geschehens. Der Kapitalismus ist sein eigener Totengräber. Das Proletariat erscheint wie bei Marx als Heiland der Welt, als Träger der weltgeschichtlichen Befreiung. ${ }^{5}$

Nun wendet sich Becker zeitweilig auch gegen die Verhältnisse in seinem neuen schweizerischen Vaterland, obschon er zu diesem und $\mathrm{zu}$ dessen republikanischen Institutionen stets in einem ambivalenten Verhältnis steht. Man darf nicht vergessen, dass er während des schweizerischen Bürgerkrieges von 1847, des sog. Sonderbundskrieges, als Vorkämpfer auf der Seite der demokratisch-zentralistischen Fortschrittsfreunde steht. So wirkt er als Adjutant des radikalen Führers Ulrich Ochsenbein, des späteren Bundesrates, in diesem

1 Vorbote, 1869, S. $82 \mathrm{ff}$.

${ }^{2}$ Vorbote, 1870, S. $64 \mathrm{ff}$.

${ }^{3}$ Ebenda, r870, S. 66. Bezeichnender anti-proudhonistischer Gegenartikel über das Thema "Die Arbeiterbewegung, das Genossenschaftswesen und die Revolution", in: Vorbote, 1870 , S. $33 \mathrm{ff}$., $49 \mathrm{ff}$., $65 \mathrm{ff}$.

4 Ebenda, 1870 , S. I $7 \mathrm{ff}$.

5 Ebenda, 1870 , S. $33 \mathrm{ff}$. 
Feldzug mit. Erst sein bedingungsloser Einsatz für die „deutsche rothe Republik", seine Mitwirkung bei der Zeitung Revolution (später aus politischen Gründen in -Evolution umgetauft) macht ihn seinen radikalen Berner Freunden und seinen Bieler Mitbürgern so suspekt, dass er aus dem Kanton Bern verbannt wird. Das ist der Grund für seine Uebersiedlung nach Genf. Dort schliesst er sich eng an Albert Galeer und seine junge sozialdemokratische Partei an. Sie bricht indessen nach Geleers frühem Tode bald zusammen.

I 862 nimmt Becker den Faden der Galeerschen sozialdemokratischen Opposition wieder auf. Sein „Eidgenössischer Verein” versucht zwar die proletarischen Kreise Genfs zu sammeln, schliesst sich aber eng an die Radikalen James Fazys an, die damals in einem klassenkampfähnlichen Ringen mit dem liberal-konservativen Altgenfertum stehen. ${ }^{1}$ Ein Versuch, Ende 1868 eine rein proletarischklassenbewusste Partei zu bilden, die „République démocratique et sociale"," scheitert am Widerstand Fazys. Dieser weist in der entscheidenden Wahlversammlung nach, dass eine derartige Gründung nur die radikale Partei, die sich damals in der Opposition befindet, schwächen und damit die liberal-konservative Mehrheit und indirekt die Genfer Finanzoligarchie stärken würde. So bleibt die Genfer Arbeiteropposition, die sich $\mathrm{r} 87 \mathrm{x}$ als "Association politique ouvrière nationale" konstituiert, so etwas wie ein extrem linker Flügel der Radikalen. Becker segelt damals in der Genfer Politik durchaus im Fahrwasser seines Parteigenossen Grosselin, der von den Radikalen als kandidat für den Regierungsrat aufgestellt wird und freimütig bekennt: „Le socialisme est solidement implanté à Genève grâce à la puissance des radicaux."3 Eine Erklärung der Genfer Sozialdemokraten zur Pariser Commune spiegelt die auch von Becker geteilte, zutiefst reformistische Ueberzeugung wider: „Le peuple en Suisse peut arriver paisiblement au triomphe de la représentation du travail par les moyens que les institutions républicaines lui offrent". ${ }^{4}$ In der Tat gewinnt die Genfer Sozialdemokratie dank dieser Allianzstellung in den Jahren der radikalen Mehrheit von I 870-79 bei den Grossratswahlen jeweilen bis zu 10-15\% der Sitze des kantonalen Parlamentes. 1877 gründet Becker eine neue Zeitung, den Précurseur, der als eine Art sozialdemokratischen Genfer Parteiorgans anzusehen ist.

1 Vgl. Beckers Wahlbroschüre Der Eidgenössische Verein an die Fortschrittsmänner in Genf, Genf I 862 und die ebenfalls von Becker stammende Analyse Ein Blick in das Parteiwesen des Kantons Genf von einem Eidgenossen, Genf I 864 .

2 Vorbote I868, S. I 54.

Soldarité vom 16. Juli 1870; Egalité, No. 23 vom Ir. Juni 1870; No. 2 vom 2. Februar $187 x$.

4 Egalité, No. 6 vom 8. April I870. 
In seinem damaligen Schrifttum finden sich nun freilich auch Zeugnisse einer betonten klassenmässigen Antipathie gegen die demokratischen Institutionen, vor allem auf schweizerischer Ebene. So klagt er zum Beispiel einmal, „die auf Privatoekonomie beruhende Kapitalmacht beherrsche alle Verhältnisse, alles Gemeinsame in seine Atome auflösend". Deswegen blieben selbst die erweiterten Volksrechte in der Schweiz unfruchtbar. ${ }^{1}$ Und doch kämpft Becker 1872 und 1874 - allerdings im Gegensatz zu seinen Genossen - leidenschaftlich für die Revision der Schweizer Bundesverfassung, die - neben zentralistischen Postulaten - ja eben diese Volksrechte ausbauen will. Er wirkt damals und später als regelmässiger Korrespondent des Kampforgans der demokratischen Partei, des Winterthurer Landboten. So gehört er zusammen mit dessen Redaktor, Salomon Bleuler, der Spitzengruppe der sozial fortschrittlichen demokratischen Linksopposition an, die $1873 \mathrm{im}$ sog. Arbeiterbund ihre neue politische Form findet. Diese halb gewerkschaftliche, halb parteimässige Organisation erklärt übrigens Beckers Précurseur $1877 \mathrm{zu}$ ihrem offiziellen französischen Organ. Der Arbeiterbund betätigt sich intensiv an der schweizerischen Politik, stellt bei den Wahlen in Verbindung mit dem Grütliverein, einem nationalgesinnten Arbeiterverein, eigene Kandidaten auf und erlebt 1877 die Genugtuung, das schweizerische Fabrikgesetz in der Volksabstimmung durchzubringen. So bleibt Becker also auch als Anhänger Marxens für die Fortentwicklung der schweizerischen Demokratie offen. Er empfindet diese mithin durchaus nicht nur als Klassenstaat.

\section{JAMES GUILLAUME UND DIE JURASSISCHE DOKTRIN}

In einer Bestimmung seines eigenen Standorts sieht Guillaume die Wurzel des jurassischen Sozialismus bei Proudhon. ${ }^{2}$ Diesem gegenüber sei sein eigener Sozialismus durch drei Züge bestimmt: Er ist einerseits "socialisme absolument scientifique et expérimental" und anderseits ,,socialisme absolument populaire, vivant, agissant et non plus abstrait et doctrinaire". 3 Als drittes Kennzeichen nennt er die antiautoritäre Linie. Guillaume hebt seine eigene Position vor allem

1 Vorbote, 1869, S. 22f. Anlässlich eines Zornausbruchs Beckers, der auf eine Klage betreffend Beschlagnahmung seiner Pakete durch die französischen Zollbehörden von der Bundeskanzlei eine gleichgültige Antwort erhalten hatte.

2 Solidarité vom 20. August I870; James Guillaume, L'Internationale, Documents et Souvenirs (1864-1878), Paris 1907ff., Bd. II, S. 72.

3 Ebenda. 
ab vom Sozialismus eines Marx, eines Blanqui und eines Tschernyschewsky. Das Schwergewicht seiner Lehre liegt demnach in der eigentümlichen Verbindung von rationalen mit emotionalen Elementen mit einem neuen Organisationsprinzip. Auch wenn man eine Doktrin nicht wie einen Kuchen in Stücke teilen kann, so lässt sich die Ansicht vertreten, derjenige Teil, den Guillaume als ,populaire, vivant, agissant" bezeichnet, sei das eigentlich Bakuninsche Erbteil, von dem er übrigens auffallenderweise gar nicht spricht. Wenn Nettlau die Beziehung Guillaumes zu Bakunin mit der Formel festhält, dieser sei mehr zerstörend, jener mehr aufbauend gewesen, dann visiert er offenbar etwas ähnliches wie Guillaume selbst. ${ }^{1}$ Präzisierend darf man sagen, dass Guillaume und die Jurassier bezeichnende Lehrstücke Bakunins als Grundanschauungen in ihre Lehre verweben, aber sie immer mit einem eigenen Erlebniswert versehen. Wir meinen vor allem den Glauben an die Revolte als Bewegungsprinzip des Volkes, die Erstickung des Volksmässigen durch den Staat und dessen Stützen, Kirche und Armee, und schliesslich das Naturgesetz der Solidarität als Antithese zur staatlichen Zwangsgemeinschaft. Guillaumes Hauptleistung besteht mithin in der wissenschaftlichen Analyse der kapitalistischen Wirtschafts- und Sozialordnung und in einer Theorie der Träger und Uebergangsformen der kommenden Gesellschaft.

Was wir jurassische Doktrin nennen, ist indessen nicht ausschliesslich das persönliche Werk Guillaumes. Bigler hebt mit Recht hervor, dass sich im Jura eine Schicht führender Mentoren herausgebildet habe, welche als Gleichberechtigte an ihrer Formulierung beteiligt gewesen seien.2 So beruht also in der jurassischen Bewegung nicht nur das soziale, sondern auch das geistige Leben auf dem Prinzip der Kollektivität. Auch wenn man Guillaumes intellektuelle Spitzenposition nicht verdunkeln will, erscheint die jurassische Doktrin letztlich als Ausfluss einer intimen geistigen Gemeinschaft, die man am ehesten mit dem Zweibund von Marx und Engels und ihrem gegenseitigen Geben und Nehmen vergleichen könnte. Bigler bezeichnet sie als „revolutionäre Intimität". ${ }^{3}$ Als Mitglieder dieses engsten Führungskollektivs erwähnt er Guillaume, Spichiger und Schwitzguébel, später auch Pindy, Brousse und Kropotkin. Thomann bezieht in seine weitläufige Analyse der jurassischen Doktrin ebenfalls Bakunin, Brousse und Kropotkin als Kronzeugen ein, leider so systemlos, dass eine fehler-

\footnotetext{
1 Max Nettlau, Der Anarchismus von Proudhon zu Kropotkin. .., Berlin 1927, S. 220.

${ }^{2}$ Rolf R. Bigler, Der Libertäre Sozialismus in der Westschweiz, Köln/Berlin ${ }_{196}$, S. 2 IIff.

Ebenda, S. 214 f.
} 
hafte Optik entsteht. ${ }^{1}$ Wir vertreten demgegenüber die These, dass man zu den eigentlichen Trägern der jurassischen Doktrin nur Guillaume, Schwitzguébel und Spichiger zählen darf, aber dass man ihre Beiträge als ein Ganzes betrachten muss. Rein quellenmässig gesehen, besitzt dabei allerdings Guillaume ein Uebergewicht. Denn sein Monumentalwerk über die Internationale und sein Mémoire von I 873 bieten sich als wichtigste Untersuchungsgrundlage an. I876 fasst er zudem seine Grundansichten in der lehrhaften Schrift Idées sur l'organisation sociale zusammen. ${ }^{2}$ Schliesslich tritt er uns am häufigsten in den von ihm redigierten Zeitungen entgegen, im Progris, in der Solidarité und im Bulletin. Das hauptsächlichste Sprachrohr Schwitzguébels und Spichigers ist der Almanach du peuple (1871-75). Auf die zahlreichen „zugewandten Orte”, die sich in diesem Periodicum äussern, gehen wir nicht ein. ${ }^{3}$ Die wichtigsten Artikel Schwitzguébels gibt Guillaume 1908 in einem Sammelband heraus. 4

Thomann macht mit Recht darauf aufmerksam, dass die Empfangsbereitschaft der Jurassier für Bakunins Grundanschauungen von Revolte und Staat in ihrem eigenen Erlebniskreis begründet sei. ${ }^{5}$ Es ist nicht nur der Wahlmisserfolg von 1868 , der Guillaumes negatives Verhältnis zum Staat hervorruft, sondern ebensosehr die Begegnung mit dem autoritären Staat der Berner und Neuenburger und ihrer Staatskirche. ${ }^{6}$ Die Feindschaft gegen die Kirche als Trabantin des Staates basiert u.a. auf Guillaumes persönlichem Zusammenstoss mit der protestantischen Geistlichkeit des Kantons Neuenburg. Seine Verfemung als Atheist führt den religiösen in einen sozialen Konflikt über. ${ }^{7}$ Auch Schwitzguébel empfindet die Auseinandersetzung mit der Kirche als Klassenkampf. 8 Dem jurassischen Antimilitarismus liegt die Empörung über das Truppenaufgebot anlässlich des Lausanner Bauarbeiterstreiks zugrunde. ${ }^{9}$ Auch die für die Jurassier so

\footnotetext{
1 Charles Thomann, Le mouvement anarchiste dans les Montagnes neuchâteloises et le Jura bernois, La Chaux-de-Fonds I947, S. I4I-233.

2 James Guillaume, op. cit., III, S. 240f.; Nettlau, Anarchismus, S. $220 f f$. Auch Greulich stützt sich in seinem Anarchismus-Artikel in Richters Jahrbuch für Sozialwissenschaft, Bd. I, Teil I, zur Hauptsache auf diese Schrift. Andere grössere und kleinere theoretische und geschichtliche Werke nennt Bigler, S. 340.

3 So etwa Elisée Reclus, Guesde, Bakunin, Madame Léo, Malon, Lefrançais, Brousse etc.

4 Adhémar Schwitzguébel, Quelques écrits, Paris 1908, mit einer „Préface d'un ami” (Guillaume).

5 Thomann, Mouvement anachriste, S. I42ff.

6 Ebenda.

'La vie ouvrière, Paris 1914, darin A. Spichiger, Souvernirs du Locle, S. 219.

${ }^{8}$ Quelques écrits, S. 133.

- Antony Babel, La Première Internationale, ses débuts et son activité à Genève, in: Mélanges d'études économiques et sociales offerts à M. William Rappard, Genève 1944, S. 345 .
} 
typische Sicht des Klassenkampfes ruht in einem Urerlebnis, im Zusammenstoss mit dem damaligen Radikalismus. Die Einsicht in die Identifizierung des bestehenden Staates mit der radikalen Partei provoziert den jurassischen Ideologieverdacht gegen die Bourgeoisie als herrschende Klasse. ${ }^{1}$ Der Klassenkampf wird zur alles erklärenden Grunderscheinung des Zeitalters: „L'antagonisme entre le capital et le travail a pris le caractère d'une guerre sociale permanente". ${ }^{2}$

Aber nun besitzt der Klassenkampf in der jurassischen Doktrin eine ganz andere Funktion als etwa bei Marx, bei dem er wesentlich politisch bestimmt ist. ${ }^{3}$ Er wird vorwiegend wirtschaftlich erlebt, als gewerkscbaftlicher Klassenkampf. Im Nachdenken über die Ziele, die organisatorischen Formen und den organisatorischen Rabmen dieses gewerkschaftlichen Klassenkampfes liegt der bedeutendste Beitrag der Jurassier zur sozialistischen Doktrin in der Schweiz. Das autochthon Jurassische haben wir dabei in der seltsamen Verbindung von Realität und Utopie zu erblicken. Man kann sie nicht nur als das eigentliche Rückgrat der jurassischen Doktrin bezeichnen, sondern auch als ihren original schweizerischen Grundzug. Die intime Befassung mit den Angelegenheiten des eigenen kleinen Gemeinwesens bewirkt einerseits die für den Schweizer so typische Grundhaltung, die enge Bezogenheit zu den Realitäten des Lebens, aber anderseits auch den Versuch, aus dieser kleinen Gemeinschaft etwas möglichst Vollkommenes zu machen. Die sprichwörtliche schweizerische Qualitätsarbeit, die sich in besonderer Weise auf dem Gebiete der Uhrenfabrikation ausdrückt, ist nur ein Sonderaspekt eines allgemeinen Vollkommenheitsstrebens, das sich im ganzen öffentlichen Leben manifestiert. Ich erinnere etwa an Zwinglis Versuch, die göttliche Gerechtigkeit in einem Idealstaat hienieden schon zu verwirklichen, oder an Rousseaus politischen Idealstaat, in dem ein vollkommenes Aufgehen des Einzelnen im Ganzen angestrebt wird. So gehen auch die Jurassier immer konsequent von der sozialen Realität ihrer Situation aus und suchen nach Möglichkeiten, sie schon im Hier und Jetzt so vollkommen als möglich umzugestalten. Aber was auch immer dabei an Neuem in concreto entworfen wird, das darf seinen Sinn nie in sich selbst finden, sondern hat Gültigkeit nur als Hinweis auf die gesuchte Zukunftsgesellschaft. So sind alle jurassischen Aktionen und Organisationen immer beides zugleich: Instrumente der Gegenwartsbewältigung und Vorformen des kommenden Milleniums. Sie sind also immer als

\footnotetext{
${ }^{1}$ Quelques écrits, op. cit., S. 66ff.; Progrès, No. 7 vom 3. April I869.

2 Ebenda, S. 68.

- Biglers Meinung (S. 16I), dass sich „der ideologische Fremdkörper der marxistischen Klassenkampftheorie in den jurassischen Anarchismus gezwängt" habe, verkennt, dass es eben einen autonomen gewerkschaftlichen Klassenkampfbegriff gibt.
} 
eine Art Stufenfolge zu verstehen, in welcher der Kampf gegen das Alte, das sichtbare Zeichen der Neugestaltung und der utopische Zukunftsentwurf ineinander übergehen.

Betrachten wir diese Stufenfolge zunächst im Rahmen der Kampfformen. Realer Ausgangspunkt der jurassischen Doktrin ist der gewerkschaftliche Klassenkampf, der Streik. Erst der Streik lässt den Arbeiter die Notwendigkeit der Organisation drastisch fühlen, erst der Streik schafft also das Solidaritätsbewusstsein, welches die Grundlage der Zukunftsgesellschaft ist. ${ }^{1}$ Aber der Streik ist zur gleichen Zeit auch schon der erste Schritt in die Zukunftsgesellschaft. Wenn die kämpfende Gewerkschaft die Löhne erhöht und die Arbeitszeit reduziert, dann transformiert sie dadurch auch bereits die bestehende kapitalistische Gesellschaft. Indem sie die „faits économiques” zersetzt, legt sie und das ist die zweite Stufe - die ersten Bausteine zu einer neuen Wirtschaft. ${ }^{2}$ Hier stossen wir auf eine entscheidende Gabelung der jurassischen Doktrin. Wer dieses Ziel auf politischem Wege zu erreichen sucht, stützt den Staat als Institution und befestigt damit ein wichtiges Herrschaftsinstrument der kapitalistischen Klasse. ${ }^{3}$ Die echte „Politique du prolétariat" gipfelt demgegenüber in der Summierung des gewerkschaftlichen Kampfes, im Generalstreik, mit dem allein die bestehende Ordnung zerstört werden kann. ${ }^{4}$ Die dritte Stufe des gewerkschaftlichen Klassenkampfes ist also identisch mit der sozialen Revolution. Hier kommt freilich noch einmal die Bakuninsche Wurzel der jurassischen Doktrin zum Vorschein. Vorstellungsinhalt der sozialen Revolution ist die "action spontanée des masses”, eine Umschreibung der Bakuninschen „Revolte”. Das Urbild der sozialen Revolution erlebt der Jurassier in der Pariser Commune. Sie vollzieht sich in einem doppelten Akt: in der Abschaffung der Unterdrückungselemente, also in der Demolierung des Staates, der Armee, der Kirche, des Polizei- und Justizapparates, ${ }^{5}$ und in der Vergesellschaftung der Produktionsmittel. 6

Aber die Neuordnung der Wirtschaft wächst nun aus den von den Gewerkschaften bereit gestellten Organisationsformen heraus, insbesondere aus der Coopération. Es lässt sich nämlich auch in organisato-

${ }^{1}$ Quelques écrits, op. cit., S. zff., 17 ff, 88ff; Progrès, No. 23 vom I3. Dezember 1869.

Bulletin de la Fédération jutassienne vom 28. Februar 1875 .

$\checkmark$ Ebenda.

- Quelques écrits, S. 70, 9off.

5 Die politische Seite der Revolution wird von Guillaume schon in den revolutionären Erhebungen vom Herbst 1870 gesehen; vgl. die Sondernummer 22 der Solidarité 1870 und das Manifest anlässlich des Lyoneraufstandes, Mémoire, S. 36ff.

- Quelques écrits, S. 4off. 
rischer Hinsicht dieselbe Stufenfolge beobachten. Die Jurassier gehen wie alle andern Internationalen von den bestehenden Formen aus, vor allem von den beruflichen Unterstützungskassen der Uhrenarbeiter, also vom Mutualisme. Aber deren Tätigkeit hat nur dann einen Sinn, wenn sie sich zu Instrumenten des wirtschaftlichen Kampfes umwandeln, wenn sie als Widerstandskassen in den Kampf gegen die kapitalistische Ordnung gestellt werden. Aber auch die streikende Gewerkschaft ist nur Mittel zum Zweck. Sie hat ihre Mitglieder im Geiste des Sozialismus zu schulen und sie hiemit zur möglichen Basis der kommenden Gesellschaft auszuweiten. „Les corps de métier”, erklärt Schwitzguébel 1870 , haben nicht nur das Ziel, „de contrebalancer la puissance du capital ... mais elles préparent l'organisation générale du prolétariat, elles habituent les ouvriers à identifier leurs intérêts à pratiquer la solidarité et à agir en commun pour l'intérêt de tous. En un mot elles sont la base de l'organisation sociale à venir, puisque les associations ouvrières n'auront qu'à prendre la direction des entreprises industrielles et agricoles, alors que l'outillage, le sol, les mines etc. leur seront concédés par la collectivité qui en sera devenue propriétaire au moyen de la liquidation sociale". ${ }^{1}$

Die der „Solidarité" entsprechende Organisationsform ist die Coopérative. Es ist eines der Hauptanliegen der jurassischen Doktrin, richtige und falsche kooperative Formen auseinanderzuhalten. Das Urbild der jurassischen „Coopérative" ist das "Atelier coopératif”, eine Gemeinschaft, in der die Produktionsmittel den Arbeitern gehören, in der also die Gesetze des Kapitalismus aufgehoben sind. In die Irre führen dagegen alle jene Genossenschaften, die bloss als Palliativmittel gegen den bestehenden Kapitalismus dienen, sei es, dass sie dessen Hilfsmittel in Anspruch nehmen wie die eines Coullery, sei es, dass sie sich durch staatliche Mittel bestechen lassen, wie Lassalle es vorschlägt.2 August Spichiger begründet in einem instruktiven Artikel ausführlich, unter welchen Umständen das Atelier coopératif als „moyen d'émancipation” dient, und wann sie dieser den Weg versperrt. ${ }^{3}$ Als Muster erscheint das Atelier Coopératif der Graveurs von Le Locle, dessen Statuten uns über die jurassische cooperative Praxis Auskunft erteilen. Ihr wirtschaftliches Grundprinzip ist der

1 Rapport der Sektion Courtelary vom 29. August 1870, in: La Première Internationale, Recueil de documents, publié sous la direction de Jacques Freymond, 2 Bände, Genf. 1962, I. S. I 23 f.

Quelques écrits, op. cit., S. Io; Solidarité, No. 7 vom 21 . Mai 1870.

3. Spichiger, La coopération de production comme moyen d'émancipation, in: Almanach du peuple pour 1875, S. 28-36.

- Mémoire présenté par la Fédération jurassienne de l'Association Internationale des Travailleurs à toutes les Fédérations de l'Internationale, Sonvillier (1873) (p. James Guillaume). 
gemeinsame Besitz an Produktionsmitteln. Er erlaubt, das sozialistische Prinzip der „Egalité des salaires” zu verwirklichen. Die Remuneration erfolgt ausschliesslich nach dem Grundsatz der aufgewendeten Arbeitszeit. ${ }^{1}$ Der Reinertrag, der über diese hinausgeht, muss dem Atelier als "capital social" zufliessen. Doch ohne den Geist der Solidarité lässt sich ein derartiger Wirtschaftskörper nicht führen. Entscheidend ist also ihr sozialistischer Geist, der alle seine Mitglieder so sehr beseelt, dass sie nicht mehr an ihren persönlichen Nutzen, sondern nur mehr ans Ganze, an die Steigerung der Produktion denken. ${ }^{2}$ Die Coopérative ist gleichzeitig auch als cercle d'études sociales gedacht. Damit ist die höchste Stufe in der Stufenreihe der Organisationsformen erreicht. So wie der gewerkschaftliche Klassenkampf in die soziale Revolution ausmündet, so entwickelt sich aus der Gewerkschaft als sozialer Körper die kollektivistische Wirtschaftsform der Gegenwart und der Zukunft heraus. Das Atelier coopératif, aus der kämpfenden Gewerkschaft herauswachsend, wird zum organisatorischen Baustein der Zukunftsgesellschaft, der Commune sociale. Mit diesem Stichwort berühren wir die beiden restlichen Aspekte der jurassischen Doktrin, den Organisationsrahmen und die Zukunftsgesellschaft.

Das organisatorische Grundprinzip des Atelier coopératif besteht darin, dass ihre Leitung nicht in den Händen eines Einzelnen, sondern einer Kollektivität ruht. ${ }^{3}$ Guillaume nennt diese Abneigung gegen die zentrale Leitung jeglicher Gruppe, den „esprit antiautoritaire”, „le trait essentiel" der jurassischen Doktrin. ${ }^{4}$ Bigler stellt sein ausgezeichnetes Kapitel über die „Organisation der Fédération jurassienne” unter das Motto der Autoritätsfurcht. ${ }^{5}$ Zwar ist dieser Grundzug der Doktrin nicht autochthon jurassisch, sondern geht auf Impulse von Proudhon und Bakunin zurück. Aber er erhält doch im Milieu des Jura eine eigenartige Verbindung mit dem Boden, auf dem er weiterentwickelt wird. - Gleich der Einzelgruppe, die ausschliesslich vom Geist der Solidarité geleitet werden soll, kann auch die grössere Einheit, der Bund der Gruppen, nur auf der Stufe weltweiter brüderlicher Gesinnung gedeihen. Bakunins „Fraternité mondiale” erhält hier eine ganz neue Bedeutung. Der Russe denkt an eine Geheimarmee, die im Dienste der Revolte steht. Bei den Jurassiern werden Revolution und Organisation gleichsam identisch. „La révolution, c'est l'organisation quotidienne pendant des années!"6

1 Spichiger, op. cit., S. 35 ; Mémoires, S. 67.

2 Spichiger, op. cit., S. 34 .

${ }^{3}$ Mémoire, S. 67.

4 Solidarité vom 20. August I87o; Guillaume, Bd. II, S. $72 \mathrm{f}$.

s Bigler, Libertärer Sozialismus, S. 204ff.

${ }^{6} \mathrm{La}$ vie ouvrière I914; Darin, Maxime Leroy, S. 226. 
Das Endziel der Organisation ist die Aussöhnung von Liberté und Solidarité. Die Autonomie der Gruppe bedarf als Egänzung der intensiven Verflechtung in einem immer grösser werdenden Rahmen, der schliesslich die Welt umspannt. So wird das organisatorische Aufbauprinzip von der jurassischen Foederation übertragen auf die ganze Internationale. Ihr einziges Band ist die "Solidarite”". Aber auch in diesem Falle ist die reale Organisation im Hier und Jetzt die Präformation der Zukunftsgesellschaft. Man stellt sie sich als einen Bund freier, aber intensiv untereinander verbundener „Communes” vor, als „Fédération des libres associations" auf weltweiter Ebene. So wie die Pariser Commune für die Jurassier Urbild der sozialen Revolution ist, so ist sie für sie auch sichtbares Zeichen ihres Foederalismus. „La révolution de Paris est fédéraliste", ruft Guillaume im April i 87 I aus. ${ }^{1}$ Er nimmt diesen Anlass wahr, um den weltweiten Foederalismus vom bundesstaatlichen Foederalismus der Schweiz oder der USA abzuheben. Sein wesentlicher Gehalt besteht in der Ersetzung der Staaten und der Gesetze durch den Vertrag. Die Verträge, welche die einzelnen Communes untereinander verbinden, sollen synallagmatisch sein, d.h. die Gruppen durch den intensiven wirtschaftlichen Austausch so stark aneinander binden, dass sie trotz ihrer Autonomie zu einer neuen Einheit verschmelzen. Die bindende Kraft ist also letztlich dieselbe utopische Grösse, welche die Einzelgruppe zusammenhält, eine mystische „force collectice”, die aus der „Fédération des groupes" beinahe automatisch hervorquillt.

So sind wir denn auf drei verschiedenen Wegen mitten im Lande der Zukunft angelangt. Wie stellen sich die Jurassier diese gegenseitige Bindung auf freiwilliger Basis im Einzelnen vor? Wir wissen, dass gerade diese zentrale Frage in den Siebzigerjahren zur grossen Auseinandersetzung zwischen Guillaume und De Paepe führt. Der Belgier, der als erster in Anlehnung an seinen Landsmann Colins den Kollektivismus befürwortet, weicht nun auch als erster von seiner utopistischen Ausprägung ab. Er vermag sich die Zukunftsgesellschaft ohne regelrechte Koordinationsinstanz, mithin ohne eine minimale staatliche Gewalt nicht vorzustellen. ${ }^{2}$ Die Jurassier dagegen bleiben ihrer Konzeption unverbrüchlich treu. In ihrem Bild der Zukunftsgesellschaft gehen sie von zwei verschiedenen Ausgangspunkten aus, von der kleinsten und der grössten Einheit, von der Commune und der Internationale. Das Bild der Commune sociale, das die Jurassier entwerfen, hält sich eng an die Realität der schweizeri-

1 Solidarité, No. 2 vom 12. April 1871.

2 Vgl. De Paepes Ausführungen am Kongress der anti-autoritären Internationale in Brüssel 1874 . 
schen Gemeindeautonomie und des Atelier coopératif. Es ist auffallend, dass die Gemeinde später vom politischen Abstentionismus ausgenommen wird. Denn die politische Beteiligung am Gemeindeleben bietet die Möglichkeit der Einübung in das Leben der Zukunftsgemeinde. ${ }^{1}$ - So erscheint denn bezeichnenderweise das Modell der Zukunftsgesellschaft in der Gestalt eines jurassischen Ubrmacherdorfes. ${ }^{2}$ In ihm herrschen dieselben Gesetze, die im Atelier coopératif gelten. Alles ist rein vertraglich, nichts ist gesetzlich geordnet. ${ }^{3}$ Guillaume beschreibt hier sehr real den Uebergang von der bestehenden zur kommenden Gesellschaft. Die privaten Ateliers werden in kollektive übergeführt, seis, dass die Besitzer ihre Produktionsmittel freiwillig abgeben und ins Atelier coopératif als Mitarbeiter eintreten, seis, dass sie ganz einfach entschädigungslos enteignet werden. Aehnlich organisieren sich die Handwerker, etwa Bäcker und Metzger, und zwar in Arbeitsteilung mit den Nachbargemeinden, ferner die Bauern, die den in Gemeinbesitz übergegangenen Boden gemeinsam bewirtschaften. Schliesslich gibt es besondere Verteilerorganisationen in der Form von Konsumgenossenschaften. Die „services publics” reduzieren sich in der neuen Gesellschaft auf ein Minimum. Die Rechtssprechung wird z.B. durch ad hoc gewählte Schiedsrichter besorgt. Es ist merkwürdig, dass sich Guillaume nirgends mit den Schattenseiten derartiger Gemeinden auseinandersetzt, die ihm ja ebenso gut wie seinen Zeitgenossen von der Praxis der schweizerischen Bürgergemeinden mit ihrem Gemeinbesitz an Land her bekannt sein mussten. Greulich macht in seiner Anarchismus-Kritik mit Recht auf den engstirnigen Gemeinde-Egoismus aufmerksam. ${ }^{4}$

Ebensowenig Beachtung schenkt Guillaume dem zentralen Problem der Koordination zwischen der "Commune” und ihren Nachbargemeinden bis hinaus zu den Partnern in der weiten Welt. Es gibt kein allgemein verbindliches Prinzip, nach dem die Wirtschaftsverfassung sich richten soll. Guillaume glaubt, die Notwendigkeit des gegenseitigen Austausches werde die verschiedenartigsten Gebilde ganz einfach zur Zusammenarbeit zwingen. Die weltweite Arbeitsteilung unter den "Communes" nimmt die Internationale in die Hände. Sie wird Produktion und Konsumation im Gleichgewicht zu halten haben. Die Organisationszellen der Internationale bilden also das grosse Netz, das die Hunderte und Tausende der "Communes sociales"

1 Politique socialiste, in: Almanach du peuple pour 1874, S. I7.

2 Une commune sociale, in: Almanach du peuple pour 1871, S. $27 \mathrm{ff}$. Der Artikel stammt von Guillaume selbst.

- Hinweise auf die commune sociale und den Foederalismus in Mémoire, S. 4off.

4 Die Theorien der Anarchie, kritisch beleuchtet von H. G., in: Richters Jahrbuch für Sozialwissenschaft, Bd. I, Teil $x$, S. $16 f$. 
zusammenfasst und das Funktionieren der neuen Gesellschaft garantiert. Guillaume skizziert sie folgendermassen: „Vom Tage nach der Revolution an werden die Arbeitergruppen der gleichen Industrie das Bedürfnis haben, sich gegenseitig von Stadt zu Stadt Delegierte zu schicken, um Auskunft zu erhalten. . . D Daraus entstehen Kongresse, Bundesverträge und ständige Büros, welche zwischen den Verbandsgruppen vermitteln. Und schliesslich besteht ein Netz von Verbänden, das alle Produzenten und Konsumenten verbindet. Eine zentralisierte Statistik wird die Arbeitszeit, die Löhne und Preise und anderes festlegen". ${ }^{1}$ Das ist die Alternative zum staatliche Kollektivismus und Kommunismus, welche die Jurassier sowohl De Paepe wie auch den Anhängern des Volksstaates, z.B. einem Liebknecht, entgegenstellen, ihr letztes Wort, von dem sie um keinen Preis abweichen. $^{2}$

${ }^{1}$ Aus Guillaumes Idées sur l'organisation sociale, 1876 , zitiert bei Greulich, op. cit., S. IIf.

${ }^{2}$ Schwitzguébels Gegenreferat gegen Paepe am Kongress von Brüssel, vorgetragen am Jahreskongress der Fédération jurassienne von 1875 in Vevey (La question des services publics devant l'Internationale, in: Quelques écrits, op. cit. S. I I 7 ff.). 